\title{
Pseudo-Differential Operators, Equations and Boundary Value Problems
}

\author{
Vladimir Vasilyev ${ }^{1, a)}$ \\ ${ }^{1}$ Chair of Differential Equations, Belgorod National Research University, Studencheskaya 14/1, Belgorod 308007, \\ Russia \\ a)Corresponding author: vladimir.b.vasilyev@gmail.com
}

\begin{abstract}
We consider pseudo-differential operators in a bounded domain of Euclidean $m$-dimensional space. A boundary of the domain is a smooth surface excluding some points or smooth sub-surfaces which are singular points of the boundary. For such operators we introduce a certain operator symbol and study their Fredholm properties in Sobolev-Slobodetskii spaces.
\end{abstract}

\section{INTRODUCTION}

We study the pseudo differential equation

$$
(A u)(x)=f(x), x \in D,
$$

in the Sobolev-Slobodetskii spaces $H^{s}(D)$, where $A$ is elliptic pseudo differential operator, $D \subset \mathbf{R}^{m}$ is $m$-dimensional domain with a boundary having singularity points. Singularity points of the domain $D$ are called the points breaking the smoothness property for boundary $\partial D$. Using wave factorization concept for elliptic symbol permits to describe solvability conditions for the equation for singularities of "cone" or "wedge" type. Most of the author's results on solvability were related to plane case. Here we will consider essential multi-dimensional situation.

Our main goal is to describe Fredholm properties for the pseudo-differential equation

$$
(A u)(x)=f(x), x \in D,
$$

where $D$ is a bounded domain, $A$ is pseudo-differential operator with the symbol $A(x, \xi)$

$$
(A u)(x)=\int_{D} \int_{\mathbf{R}^{m}} A(x, \xi) u(y) e^{i(x-y) \xi} d \xi d y, \quad x \in D .
$$

Everywhere below we will suppose that this symbol is an infinitely differentiable function in $\bar{D} \times \mathbf{R}^{m}$, and it is an elliptic symbol, i.e. $A(x, \xi) \neq 0 . \forall(x, \xi) \in \bar{D} \times \mathbf{R}^{m}$.

Existence of a boundary for the domain $D$ very influences on Fredholm properties of the operator $A$ [1] even if a boundary is a smooth surface; an ellipticity of a symbol is not sufficient, and one needs some additional conditions. But there is no appropriate technique for a non-smooth boundary to describe results on Fredholmness. Here we will develop the local principle [2] in its operator form and apply it to studying solvability of model equations in canonical domains.

Such operators are defined locally by the formula

$$
u(x) \longmapsto \int_{\mathbf{R}^{m}} \int_{\mathbf{R}^{m}} A(x, \xi) u(y) e^{i(x-y) \cdot \xi} d \xi d y, \quad x \in \mathbf{R}^{m},
$$

if $D$ is a smooth compact manifold, because can use "freezing coefficients principle ", or in other words "local principle". For manifold with a smooth boundary we need new local formula for defining the operator $A$ : more precisely in

7th International Eurasian Conference on Mathematical Sciences and Applications (IECMSA-2018)

AIP Conf Proc 2037, 020028-1-020028-5; https//doi org/10 1063/15078483

Published by AIP Publishing 978-0-7354-1763-2/\$3000 
inner points of $D$ we use the formula (2), but in boundary points we need another formula

$$
u(x) \longmapsto \int_{\mathbf{R}_{+}^{m}} \int_{\mathbf{R}^{m}} A(x, \xi) u(y) e^{i(x-y) \cdot \xi} d \xi d y, \quad x \in \mathbf{R}_{+}^{m},
$$

where $\mathbf{R}_{+}^{m}$ is a half-space $\left\{x \in \mathbf{R}^{m}: x=\left(x^{\prime}, x_{m}\right), x_{m}>0\right\}$.

For invertibility of such operator with symbol $A(\cdot, \xi)$ non-depending on spatial variable $x$ one can apply the theory of classical Riemann boundary problem for upper and lower complex half-planes with a parameter $\xi^{\prime}$. This step was systematically studied in the book [1]. But if the boundary $\partial D$ has at least one conical point, this approach is not effective.

The $k$-wedge point at the boundary is such a point, for which its neighborhood is diffeomorphic to the cone $C_{a_{k}}^{m-k}=\mathbf{R}^{k} \times C_{+}^{a_{k}}$, where

$$
C_{+}^{a_{k}}=\left\{x \in \mathbf{R}^{m-k}: x=\left(x_{k+1}, \ldots, x_{m}\right), x_{m}>a_{k}\left|x^{\prime \prime}\right|, x^{\prime \prime}=\left(x_{k+1}, \ldots, x_{m-1}\right), a_{k}>0\right\},
$$

hence the local definition for pseudo-differential operator near the conical point is the following

$$
u(x) \longmapsto \int_{C_{a_{k}}^{m-k}} \int_{\mathbf{R}^{m}} A(x, \xi) u(y) e^{i(x-y) \cdot \xi} d \xi d y, \quad x \in C_{a_{k}}^{m-k} .
$$

\section{OPERATORS AND EQUATIONS IN SOBOLEV-SLOBODETSKII SPACES}

We consider the operator (1) in the Sobolev - Slobodetskii space $H^{s}\left(\mathbf{R}^{m}\right)$ with norm

$$
\|u\|_{s}^{2}=\int_{\mathbf{R}^{m}}|\tilde{u}(\xi)|^{2}(1+|\xi|)^{2 s} d \xi,
$$

where $\tilde{u}$ denotes the Fourier transform of the function $u$

$$
\tilde{u}(\xi)=\int_{\mathbf{R}^{m}} e^{i x \cdot \xi} u(x) d x
$$

and introduce the following class $S_{\alpha}$ of symbols non-depending on a spatial variable $x$ : $\exists c_{1}, c_{2}>0$,

$$
c_{1} \leq\left|A(\xi)(1+|\xi|)^{-\alpha}\right| \leq c_{2}, \quad \forall \xi \in \mathbf{R}^{m} .
$$

The number $\alpha \in \mathbf{R}$ we call an order of pseudo-differential operator $A$.

It is well-known that pseudo-differential operator with symbol $A(\xi)$ satisfying (4), is linear bounded operator acting from $H^{s}\left(\mathbf{R}^{m}\right)$ into $H^{s-\alpha}\left(\mathbf{R}^{m}\right)$ [1].

\section{OPERATOR SYMBOL}

\section{Local Operators}

A local operator in the point $x_{0} \in \bar{D}$ we call the following operator

$$
\left(A_{x_{0}} u\right)(x)=\int_{D_{x_{0}}} \int_{\mathbf{R}^{m}} A\left(x_{0}, \xi\right) u(y) e^{i(x-y) \xi \xi} d \xi d y, \quad x \in D_{x_{0}},
$$

where $D_{x_{0}}$ is one of canonical domains $\mathbf{R}^{m}, \mathbf{R}_{+}^{m}, C_{a_{k}}^{m-k}$ in dependence on a placement of the point $x_{0}$.

Obviously, the symbol of a local operator belongs to the class $S_{\alpha}$. 


\section{Local Sobolev-Slobodetskii Spaces}

We are interested in studying invertibility operator (4) in corresponding Sobolev - Slobodetskii spaces. By definition, $H^{s}\left(D_{x_{0}}\right)$ consists of distributions from $H^{s}\left(\mathbf{R}^{m}\right)$ with support in $\overline{D_{x_{0}}}$. The norm in the space $H^{s}\left(D_{x_{0}}\right)$ is induced by the norm $H^{s}\left(\mathbf{R}^{m}\right)$. We associate such operator with corresponding equation

$$
(A u)(x)=f(x), x \in D_{x_{0}},
$$

where right-hand side $f$ is chosen from the space $H_{0}^{s-\alpha}\left(D_{x_{0}}\right) ; H_{0}^{s}\left(D_{x_{0}}\right)$ is the space of distributions $S^{\prime}\left(D_{x_{0}}\right)$, which admit continuation into $H^{s}\left(\mathbf{R}^{m}\right)$. The norm in $H_{0}^{s}\left(D_{x_{0}}\right)$ is defined by

$$
\|f\|_{s}^{+}=\inf \|l f\|_{s},
$$

where infimum is chosen for all possible continuations $l$.

\section{Operator Families}

Definition 1. Local operator $A_{x_{0}}: H^{s}\left(D_{x_{0}}\right) \rightarrow H^{s-\alpha}\left(D_{x_{0}}\right)$ is called a local representative of the operator $A$ in the point $x_{0}$.

Lemma 1. Let $\varphi, \psi$ be smooth functions defined in some neighborhood $U \cap D$ of the point $x_{0} \in \bar{D}$. Then the operator

$$
T_{x_{0}}=\varphi\left(A-A_{x_{0}}\right) \psi
$$

is a compact operator $A_{x_{0}}: H^{s}(U \cap D) \rightarrow H^{s-\alpha}(U \cap D)$.

Taking into account all previous considerations $[2,3,4,5,6,7,8,9]$ we introduce the following

Definition 2. An operator symbol of the operator A is called a collection of its local representatives.

Definition 3. Operator A is called an elliptic operator if its operator symbol consists of invertible operators in corresponding Sobolev-Slobodetskii spaces.

Lemma 2. An elliptic operator $A: H^{s}(D) \rightarrow H^{s-\alpha}(D)$ has a Fredholm property.

The assertion of Lemma 2 is very general. We need finding more convenient sufficient conditions for invertibility of operator symbol.

\section{WAVE FACTORIZATION}

Below we will consider the local symbols $A\left(x_{0}, \xi\right)$ from the class $S_{\alpha}$. Since we consider fixed $x_{0}$ we will write $A(\xi)$ instead of $A\left(x_{0}, \xi\right)$ in this section. Let us denote $\xi \in \mathbf{R}^{m}$ by $(\tilde{\xi}, \tilde{\xi})$ where $\tilde{\xi} \in \mathbf{R}^{k}, \tilde{\xi} \in \mathbf{R}^{m-k}$.

Definition 4. $k$-wave factorization of symbol $A(\xi)$ is called its representation in the form

$$
A(\xi)=A_{\neq}(\xi) A_{=}(\xi)
$$

where the factors $A_{\neq}(\xi), A_{=}(\xi)$ satisfy the following conditions:

1) $A_{\neq}(\xi), A_{=}(\xi)$ are defined everywhere without may be the points $\left\{\xi \in \mathbf{R}^{m}:\left|\xi^{\prime \prime}\right|^{2}=a_{k}^{2} \xi_{m}^{2}\right\}$;

2) $A_{\neq}(\xi), A_{=}(\xi)$ admit an analytical continuation with variable $\tilde{\xi}$ into radial tube domains $T\left(\stackrel{*}{C_{+}^{a_{k}}}\right), T\left(\stackrel{*}{a_{k}}\right)$ respectively under almost all fixed $\tilde{\xi}$, which satisfy the estimates

$$
\begin{gathered}
\left|A_{\neq}^{ \pm 1}(\tilde{\xi}, \tilde{\xi}+i \tau)\right| \leq c_{1}(1+|\xi|+|\tau|)^{ \pm \mathfrak{x}_{k}}, \\
\left|A_{\cong}^{ \pm 1}(\tilde{\xi}, \tilde{\xi}-i \tau)\right| \leq c_{2}(1+|\xi|+|\tau|)^{ \pm\left(\alpha-\mathfrak{x}_{k}\right)}, \quad \forall \tau \in C_{+}^{a_{k}} .
\end{gathered}
$$

The number $\mathfrak{x}_{k}$ is called index of $k$-wave factorization.

Here $C_{+}^{a_{k}}$ is conjugate cone to $C_{+}^{a_{k}}$, and $C_{-}^{a_{k}}=-C_{+}^{a_{k}}[10,3]$. 


\section{FREDHOLM PROPERTY}

Let $D_{k}, k=1, \ldots, m-2$ be closed smooth sub-surfaces on the boundary $\partial D \equiv D_{m-1}$ such that $D_{k}$ consists of $k$-wedge points. Let us note that $D_{0}$ is a union of conical points.

Theorem. If the symbol $A(x, \xi)$ admits $k$-wave factorization for all $x \in D_{k}$ with respect to the cone $C_{a_{k}}^{m-k}$ with indices $\mathfrak{a}_{k}(x), k=0,1, \cdots, m-2$ satisfying the conditions

$$
\left|\mathfrak{x}_{k}(x)-s\right|<1 / 2, \quad \forall x \in D_{k}, \quad k=0,1, \cdots, m-1,
$$

then the operator $A: H^{s}(D) \rightarrow H^{s-\alpha}(D)$ is a Fredholm operator.

Remark 1. $\mathfrak{x}_{m-1}$ is index offactorization in Eskin's sense [1] for smoothness points.

\section{Example}

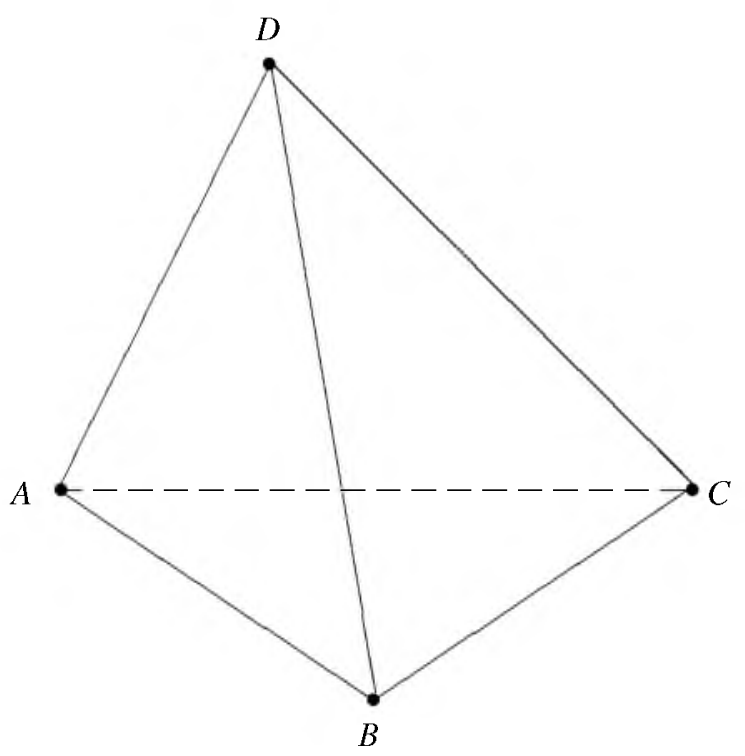

For such domain $D$ (Fig. 1) we have distinct types of local operators. Here $m=3$. One kind of local operators is of type (2) for inner points of $D$, the second one is of type (3) and it serves the points of smoothness at the boundary $\partial D$, the third kind corresponds to the 1-wedge points placed on 6 wedges $A B, B C, A C, A D, B D, C D$, and the fourth kind corresponds to the 0 -wedge points, $i$. e. conical points supported in vertices $A, B, C, D$. The latter two case are described by local operators of type (4). To obtain Fredholm property for the operator $A$ in such domain $D$ we need to have invertibility conditions for all above local operators.

\section{ELLIPTIC BOUNDARY VALUE PROBLEMS}

Let us suppose that there is a $x_{0} \in \partial D$ such that the condition (6) does not hold although the $k$-wave factorization exists. The corresponding local operator $A_{x_{0}}$ is not invertible $[1,3]$ but if, for example, $\mathfrak{x}-s=n+\varepsilon, n \in \mathbf{N},|\varepsilon|<1 / 2$, then the operator $A_{x_{0}}$ has non empty kernel. More exactly, for $x_{0} \in M_{k}$ the kernel of operator $A_{x_{0}}$ consists of the following functions [11]

$$
A_{\ddagger}^{-1}(\xi) V_{-a} F\left(\sum_{j=1}^{n} c_{j}\left(x^{\prime}\right) \delta^{(k-1)}\left(x_{m}\right)\right),
$$

where $c_{j}\left(x^{\prime}\right) \in H^{s_{j}}\left(\mathbf{R}^{m-1}\right)$ are arbitrary functions, $s_{j}=s-\mathfrak{x}+j-1 / 2, k=1,2, \ldots, n$

Here the following notations were used: $A_{\neq}(\xi)$ is an element of the $k$-wave factorization, $F$ is the Fourier transform, $\delta$ is the Dirac mass-function, and the operator $V_{a}$ is defined in the following way. We denote by $E_{a}\left(\xi^{\prime}, \xi_{m}\right)$ the 
( $m-1)$-dimensional Fourier transform $\left(y^{\prime} \rightarrow \xi^{\prime}\right.$ in distribution sense) of the function $e^{i a y^{\prime} y^{\prime} \xi_{m}}$ and introduce the operator $V_{a}$ by the following formula

$$
\left(V_{a} \tilde{u}\right)(\xi)=\left(E_{a} \star \tilde{u}\right)(\xi),
$$

where $\star$ denotes a convolution with respect to first $m-1$ variables and multiplier with respect to the last variable $\xi_{m}$.

To determine uniquely these unknown functions $c_{j}, j=1, \ldots, n$, we need some additional conditions. As a rule these conditions they give as traces of pseudo-differential operators $B_{j, x_{0}}[1,3]$. But such operators can not be arbitrary operators, these "boundary operators" should be correlated with initial operator $A_{x_{0}}$. These correlation conditions are called usually by Shapiro-Lopatinskii conditions [1].

Remark 2. It is possible to verify an invertibility of local operators for certain simplest cones. Some results of such kind are presented to publishing by the author. The two-dimensional case is considered earlier [3].

\section{ACKNOWLEDGMENTS}

This work was supported by the State contract of the Russian Ministry of Education and Science (contract No $1.7311 .2017 / 8.9)$.

\section{REFERENCES}

[1] G. Eskin, Boundary Value Problems for Elliptic Pseudodifferential Equations (American Mathematical Society, Providence, 1981), pp. 1-375.

[2] I. B. Simonenko, Local Method in the Theory of Translation Invariant Operators and Their Envelopes (in Russian) (CVVR, Rostov on Don, 2007), pp. 1-120.

[3] V. B. Vasil'ev, Wave Factorization of Elliptic Symbols: Theory and Applications. Introduction to the Theory of Boundary Value Problems in Non-Smooth Domains (Kluwer Academic Publishers, Dordrecht-BostonLondon, 2000), pp. 1-192.

[4] V. B. Vasil'ev, Trans. Moscow Math. Soc. 59, 65-93 (1998).

[5] V. B. Vasilyev, J. Math, Sci. 230, 175-183 (2018).

[6] V. B. Vasilyev, J. Math. Sci. 234, 397-406 (2018).

[7] V. B. Vasil'ev, Differ. Equ. 51, 1113-1125 (2015).

[8] V. B. Vasilyev, "Asymptotical analysis of singularities for pseudo differential equations in canonical nonsmooth domains," in Integral Methods in Science and Engineering. Computational and Analytic Aspects, edited by C. Constanda and P. J. Harris (Birkhauser, Boston, 2011), pp. 378-390.

[9] V. B. Vasilyev, "Pseudo-differential operators on manifolds with a singular boundary," in Modern Problems in Applied Analysis, edited by P. Drygas and S. Rogosin (Birkhauser/Springer, Cham, 2018), pp. 169-179.

[10] S. Bochner and W. T. Martin, Several Complex Variables (Princeton Univ. Press, Princeton, NJ, 1961), pp. $1-216$.

[11] V. B. Vasil'ev, Sib. Elektron. Mat. Izv. 13, 1129-1149 (2016). 